\title{
The Substellar Population in IC 348
}

\author{
K. L. Luhman \\ Harvard-Smithsonian Center for Astrophysics, 60 Garden Street, \\ Cambridge, MA 02138
}

\begin{abstract}
I present the latest results in a continuing effort to spectroscopically identify the substellar members of the young cluster IC 348 . I have combined new images at $I$ and $Z$ with IR photometry from previous studies and from 2MASS and selected candidate members of the cluster across a larger area and at lower masses than probed in previous surveys. After observing these candidates with spectroscopy, the latest census for IC 348 contains 234 members with spectral types, 22 of which are later than M6 and thus are probably substellar. Individual masses of cluster members are estimated by combining their positions on the HertzsprungRussell (H-R) diagram with theoretical evolutionary models. From an extinction-limited sample of members $\left(A_{V} \leq 4\right)$, I construct an IMF for IC 348 that should be complete down to $0.02 M_{\odot}$. This IMF is compared to a similarly derived mass function for Taurus by Briceño et al. (2002); these data provide clear evidence for a variation of the IMF with star-forming conditions.
\end{abstract}

\section{Introduction}

The young cluster IC 348 is well-suited for a survey for brown dwarfs. The cluster is young $(3 \mathrm{Myr})$, nearby $(315 \mathrm{pc})$, rich $(\sim 300$ members $)$, compact $\left(D \sim 20^{\prime}\right)$, and most of its members exhibit relatively low extinction $\left(A_{V}=0-5\right)$. As a result, substellar members of the cluster can be detected down to low masses and in large numbers through efficient observations of a small area of sky that can be performed at both optical and infrared (IR) wavelengths.

\section{Results of the Survey}

Previous studies have searched for members of IC 348 through proper motions (Fredrick 1956), H $\alpha$ emission (Herbig 1954, 1998), IR luminosity functions (Lada \& Lada 1995; Luhman et al. 1998), and optical color-magnitude diagrams (Herbig 1998; Luhman 1999). I extend the latter surveys to greater depth and to a larger area of the cluster by including new $I$ and $Z$ data. For the $16^{\prime} \times 14^{\prime}$ region in Figure 1, I plot the diagram of $I-Z$ vs. $I$ in Figure 2. I have obtained optical spectra of candidate members of IC 348 in Figure 2 and have combined the spectral types, spectral features $(\mathrm{H} \alpha, \mathrm{Na} \mathrm{I}, \mathrm{K} \mathrm{I})$, and photometry to evaluate the membership of each candidate. When measuring spectral types for candidate young brown dwarfs $(\geq M 6)$ with optical data, averages of dwarf 
and giant standards provide the best classification standards (Luhman 1999). Optically-classified young late-type objects (GY 141, known members of IC 348 and Taurus) should be used as the standards when measuring spectral types for candidates from IR spectra. In addition, it is essential that the spectra or other data provide evidence that a candidate is a young member of the target cluster rather than a field dwarf.

I use the census of spectroscopically-confirmed members of IC 348 to evaluate the survey for low-mass members by Najita et al. (2000). In that study, the $5^{\prime} \times 5^{\prime}$ center of the cluster was imaged with $H S T$ NICMOS in narrowband filters that sample the near-IR steam absorption bands. They estimated spectral types from those data and constructed an empirical H-R diagram, which is reproduced in Figure 3. At $K>14$, photometric uncertainties in the NICMOS narrowband photometry translated into very large errors in the estimated spectral types. Consequently, several field stars were mistaken for objects with spectral types of late M (Figure 3). In addition, because the spectral types from the steam data were systematically too cool (Figure 4), the masses were underestimated. These two effects both resulted in an overestimate of the number of brown dwarfs by Najita et al. (2000). In Figure 4, 28 sources have NICMOS classifications later than M6, when only 9 of these sources actually have spectral types in that range.

I have placed the members of IC 348 on the H-R diagram in Figure 5 and have used the evolutionary models to infer masses for the individual sources. I define an extinction-limited sample that should be unbiased in mass and representative of the cluster down to $0.02 M_{\odot}(\sim \mathrm{M} 9)$. This sample is now compared to a similarly complete sample for $8.4 \mathrm{deg}^{2}$ in the Taurus star-forming region (Briceño et al. 2002). Because of the vertical nature of mass tracks in the H-R diagram, the spectral types young object should be well-correlated with their masses. In addition, a spectral type is a simple, observable quantity that can be measured to good accuracy with relative ease, particularly at $\mathrm{M}$ types. Therefore, I begin with a comparison of the distributions of spectral types for IC 348 and Taurus in Figure 6. The distributions for IC 348 and Taurus are quite distinctive from each other, peaking sharply at M5 and K7, respectively. These spectral types correspond to masses of 0.15 and $0.8 M_{\odot}$ at an age of $1 \mathrm{Myr}$ for the models of Baraffe et al. (1998) and Chabrier et al. (2000). This is the first comparison of spectral type distributions between two star-forming populations that is based on samples that are complete to late spectral types and that include relatively large numbers of members. These data represent clear evidence for a significant variation of the IMF with star-forming conditions.

When the data for the samples in IC 348 and Taurus are transformed to individual masses with the evolutionary models, the IMFs in Figure 6 are produced. The IMF for Taurus peaks near $0.8 M_{\odot}$ and steadily declines to lower masses. Meanwhile, the mass function for IC 348 rises quickly from high masses down to a solar mass, rises more slowly down to a maximum at 0.1-0.15 $M_{\odot}$, and then declines quickly into the substellar regime. Meanwhile, the frequency of brown dwarfs is similar between the two star-forming regions, as quantified by $N\left(0.02 \leq M / M_{\odot} \leq 0.08\right) / N\left(0.08<M / M_{\odot} \leq 10\right)=24 / 153=0.16 \pm 0.03$ and $10 / 76=0.13 \pm 0.04$ for IC 348 and Taurus, respectively, which are roughly half of the values implied by the IMFs measured for the Trapezium Cluster (Luhman et al. 2000; Hillenbrand \& Carpenter 2000; Muench et al. 2002). 


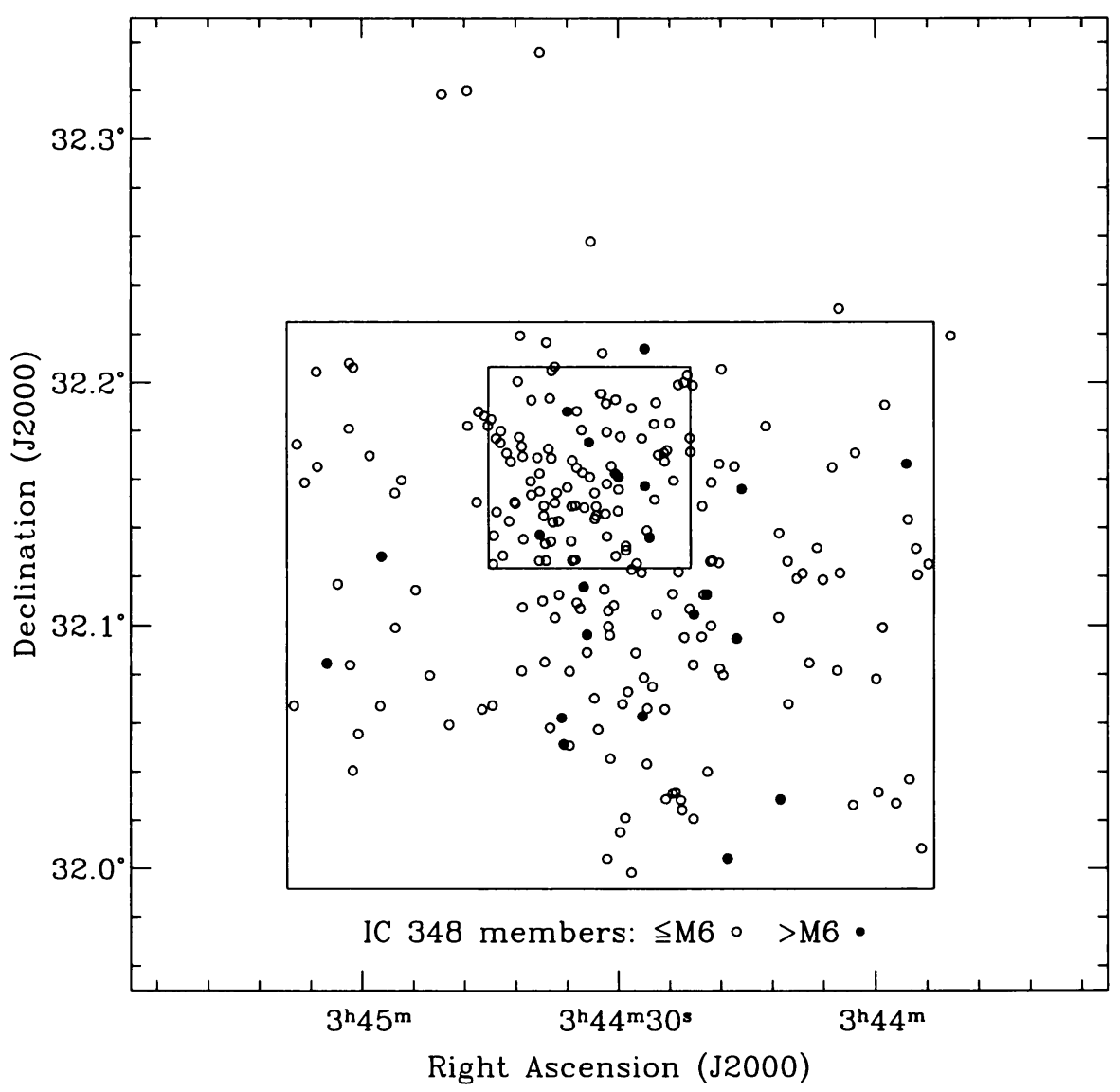

Figure 1. The spatial distribution of the 234 members of the IC 348 cluster that have measured spectral types. The 22 members with spectral types later than M6 are likely to be brown dwarfs. The analysis in this work considers the region within the large rectangle $\left(16^{\prime} \times 14^{\prime}\right)$. For reference, the IMF of Luhman et al. (1998) was measured for the area within the small rectangle $\left(5^{\prime} \times 5^{\prime}\right)$, which was closely matched by the region observed by Najita et al. (2000). 


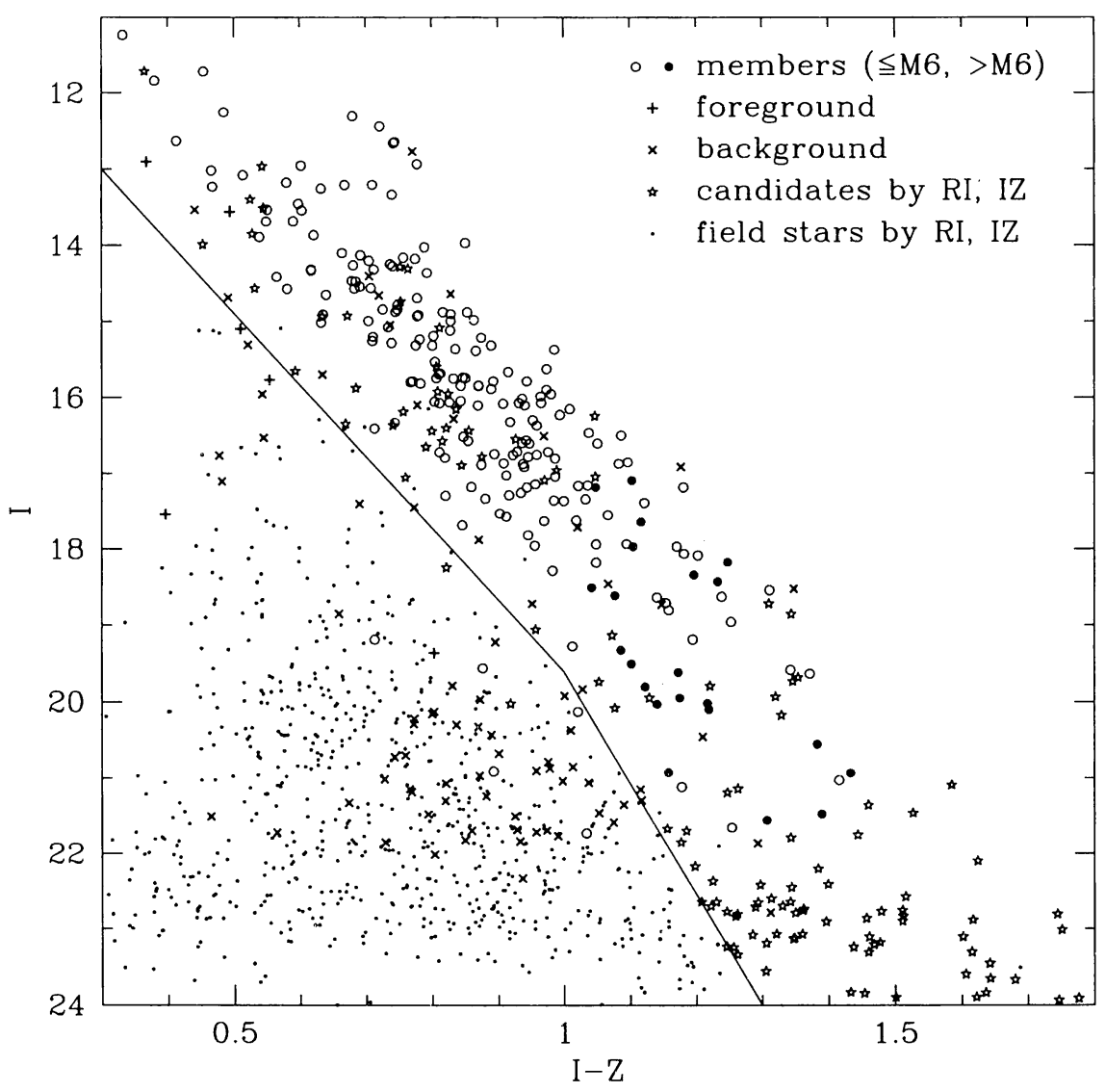

Figure 2. $\quad I-Z$ vs. $I$ for the $16^{\prime} \times 14^{\prime}$ field in the IC 348 cluster shown in Figure 1. Stars that have been spectroscopically identified as cluster members at $\leq \mathrm{M} 6$ and $>\mathrm{M} 6$ (open and solid circles) and background stars (crosses) are indicated. Foreground stars by spectroscopy or proper motion measurements are also shown (plusses). Stars that are below either of the solid boundaries here and in a similar diagram of $R-I$ vs. $I$ are probable field stars (small points). The remaining objects are candidate members of the cluster (open stars). Two objects below the boundary shows other evidence of youth and are marked as candidate as well. 


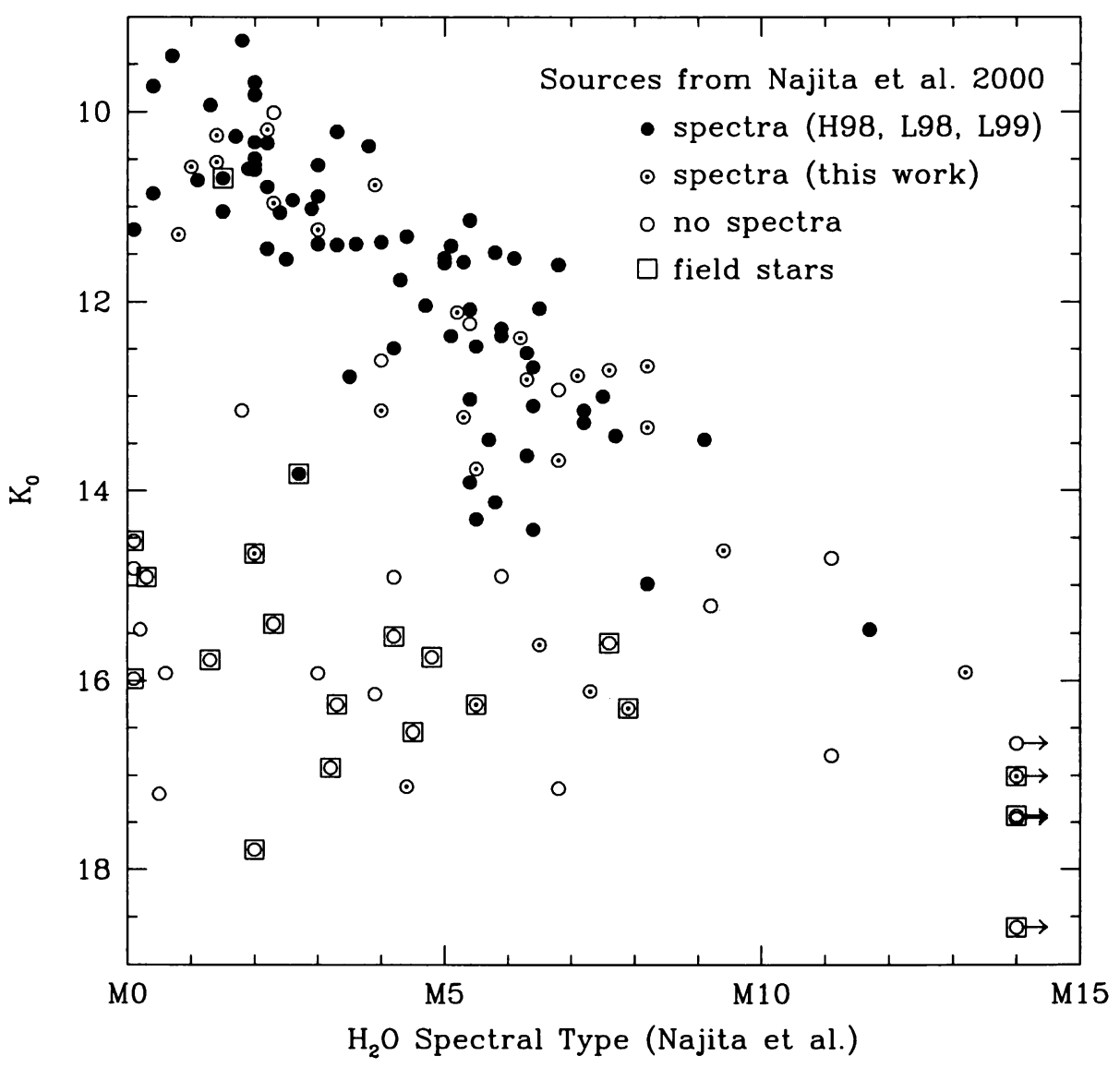

Figure 3. Spectral types estimated from $H S T$ NICMOS narrowband photometry of $\mathrm{H}_{2} \mathrm{O}$ absorption vs. dereddened $K$-band data for the core of IC 348 , as reported by Najita et al. (2000). I indicate sources that have been observed spectroscopically by Herbig (1998), Luhman et al. (1998), and Luhman (1999) (solid circles) and in this work (circled points), sources that lack spectra (open circles), and objects that are identified as foreground or background field stars by spectroscopy or the diagrams of $R-I$ vs. $I$ and $I-Z$ vs. $I$ (open squares). 


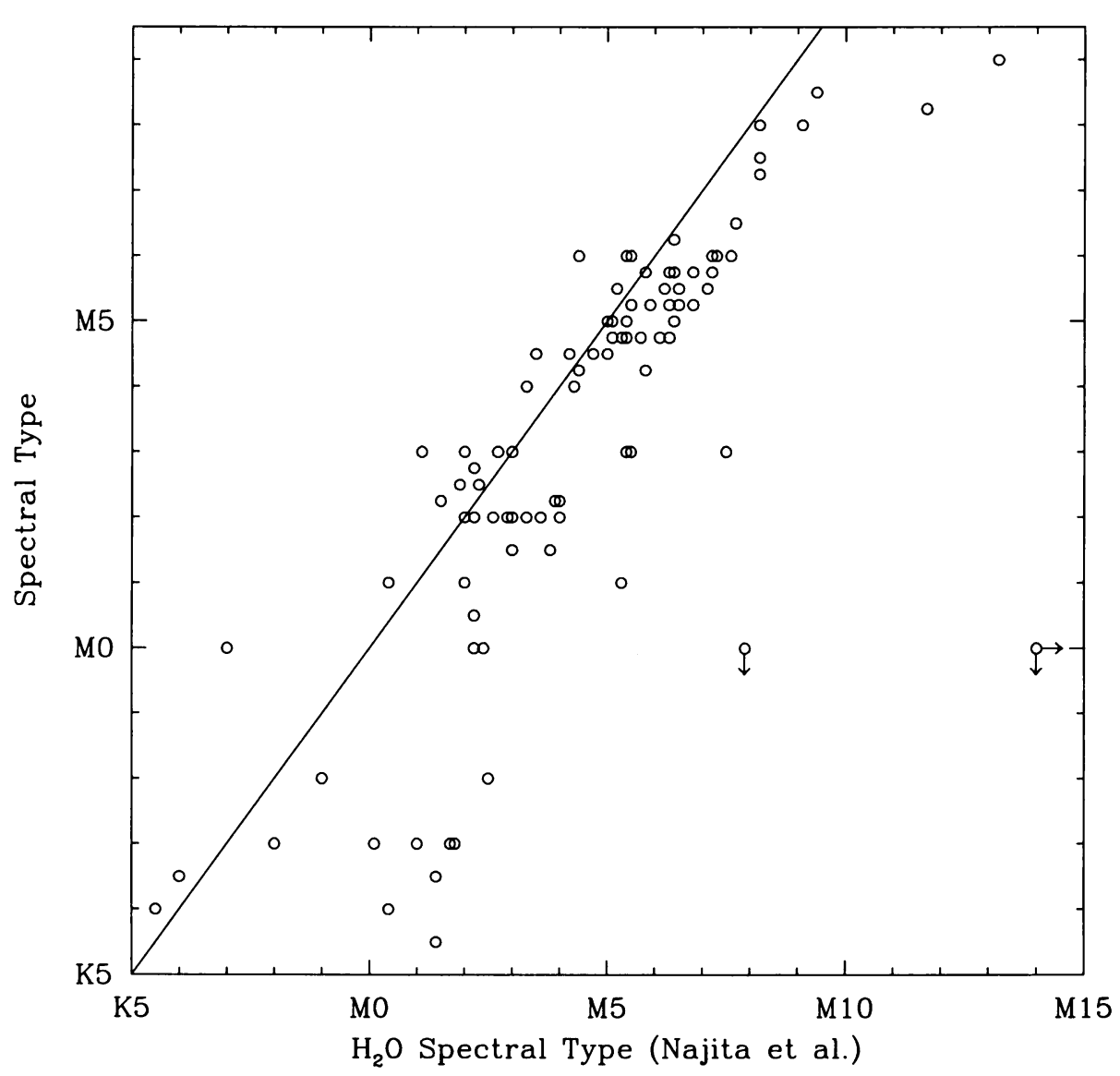

Figure 4. Spectral types estimated from HST NICMOS narrowband photometry of $\mathrm{H}_{2} \mathrm{O}$ absorption by Najita et al. (2000) vs. spectral types measured by Herbig (1998), Luhman et al. (1998), and Luhman (1999) and in this work. The typical uncertainties in the spectral types from the latter four studies are $\pm 0.25-0.5$ subclass. 


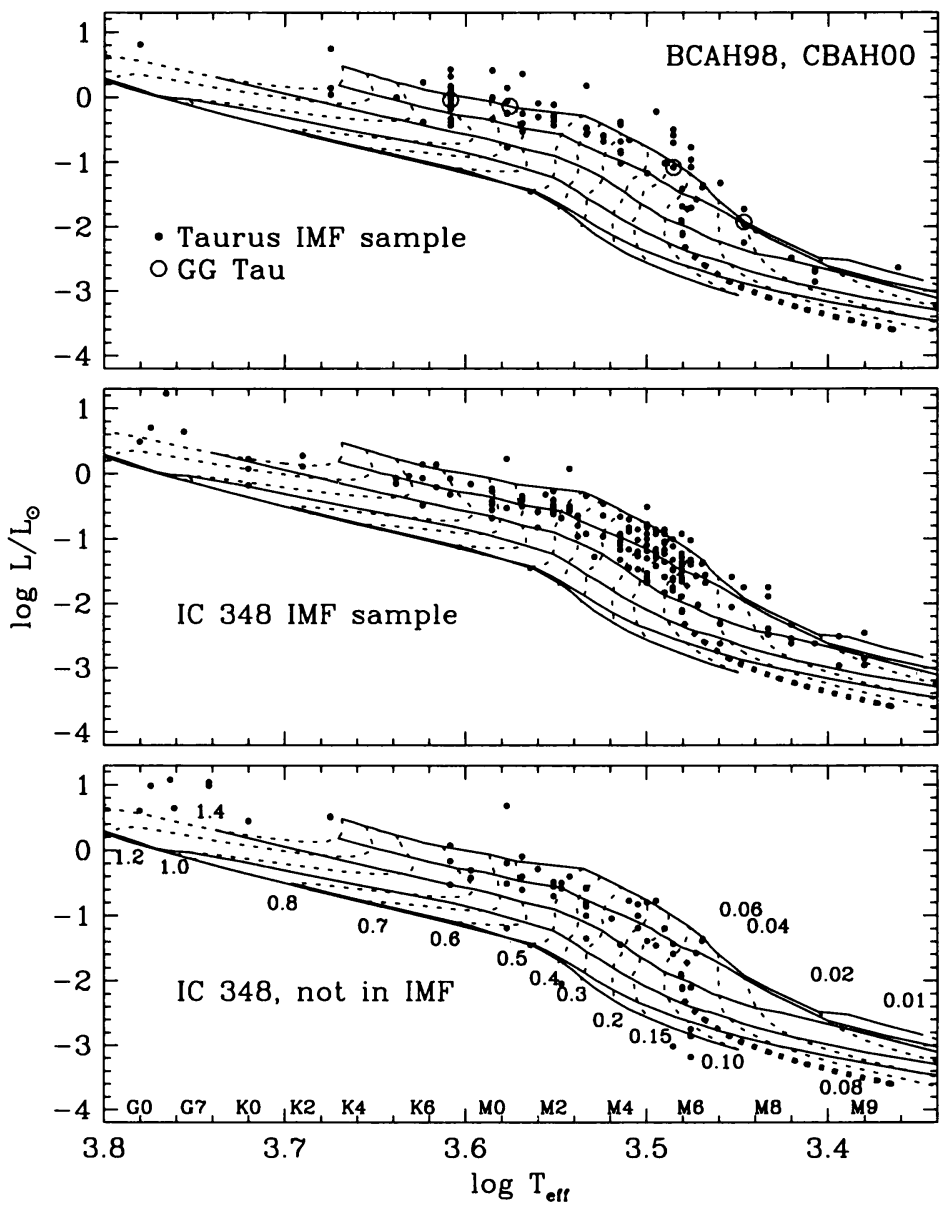

Figure 5. H-R diagrams at low masses for objects in the IMFs measured for Taurus by Briceño et al. (2002) and for IC 348 in this work (top and middle panels). Both of these IMF samples were defined by $A_{V} \leq 4$. The remaining IC 348 members are not included in the IMF because they are beyond the reddening threshold of $A_{V}=4$, are outside of the $16^{\prime} \times 14^{\prime}$ field in Figure 1, or have anomalously low luminosities for their spectral types (bottom panel). The latter sources are probably detected primarily in scattered light. The theoretical evolutionary models of Baraffe et al. (1998) and Chabrier et al. (2000) are shown, where the horizontal solid lines are isochrones representing ages of 1,3 , 10, 30, and $100 \mathrm{Myr}$ and the main sequence, from top to bottom. The $\mathrm{M}$ spectral types have been converted to effective temperatures with a scale such that GG Tau $\mathrm{Ba}$ and $\mathrm{Bb}$ fall on the same model isochrone as $\mathrm{Aa}$ and $\mathrm{Ab}$ and that the M8-M9 members of Taurus and IC 348 have comparable model ages to those of the earlier members. 

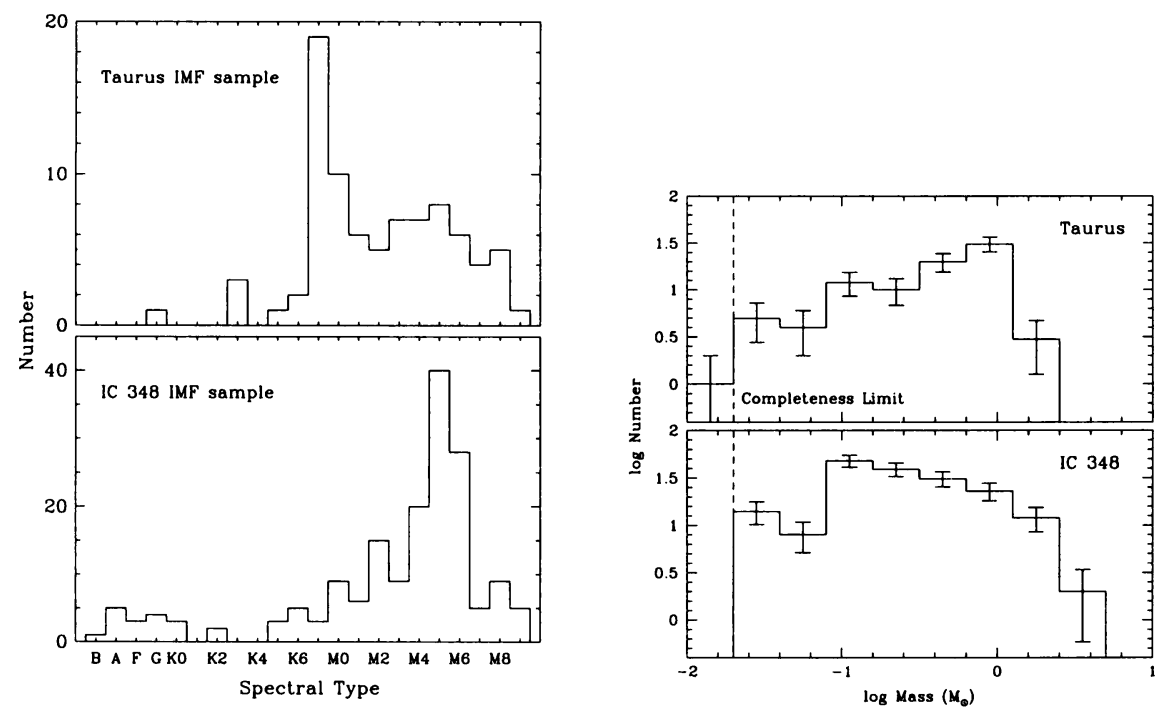

Figure 6. Left: Distributions of spectral types for objects in the IMFs measured for Taurus by Briceño et al. (2002) and for IC 348 in this work. Right: IMFs for reddening-limited samples $\left(A_{V} \leq 4\right)$ in fields within Taurus and IC 348 as inferred from the evolutionary models in the H-R diagram. Only members within the $16^{\prime} \times 14^{\prime}$ field in Figure 1 are considered for the IMF in IC 348. The Salpeter slope is 1.35.

\section{References}

Baraffe, I., Chabrier, G., Allard, F., \& Hauschildt, P. H. 1998, A\&A, 337, 403

Briceño, C., Luhman, K. L., Hartmann, L., Stauffer, J. R., \& Kirkpatrick, J. D. 2002, ApJ, submitted

Chabrier, G., Baraffe, I. Allard, F., \& Hauschildt, P. H. 2000, ApJ, 542, 464

Fredrick, L. W. 1956, AJ, 61, 437

Herbig, G. H. 1954, PASP, 66, 19

Herbig, G. H. 1998, ApJ, 497, 736

Hillenbrand, L. A., \& Hartmann, L. W. 1998, ApJ, 492, 540

Lada, E. A., \& Lada, C. J. 1995, AJ, 109, 1682

Luhman, K. L. 1999, ApJ, 525, 466

Luhman, K. L., Rieke, G. H., Lada, C. J., \& Lada, E. A. 1998, ApJ, 508, 347

Muench, A. A., Alves, J., Lada, C. J., \& Lada, E. A. 2001, ApJ, 558, 51

Najita, J., Tiede, G. P., \& Carr, J. S. 2000, ApJ, 541, 977 